User Enjoyment and Performance in Collaborative and Cooperative games in shared 3D Virtual Environments

Peer-reviewed author version

BEZNOSYK, Anastasiia; QUAX, Peter; CONINX, Karin \& LAMOTTE, Wim (2011) User Enjoyment and Performance in Collaborative and Cooperative games in shared 3D Virtual Environments. In: Proceedings of the International conference on computer graphics theory and applications. p. 302-307..

Handle: http://hdl.handle.net/1942/12159 


\title{
USER ENJOYMENT AND PERFORMANCE IN COLLABORATIVE AND COOPERATIVE GAMES IN SHARED 3D VIRTUAL ENVIRONMENTS
}

\author{
Anastasiia Beznosyk, Peter Quax, Karin Coninx, Wim Lamotte \\ Hasselt University - $t U L-I B B T$, Expertise Centre for Digital Media \\ Wetenschapspark 2,B-3590 Diepenbeek (Belgium) \\ \{anastasiia.beznosyk, peter.quax, karin.coninx,wim.lamotte\}@uhasselt.be
}

Keywords: Collaboration, cooperation, shared 3D virtual environments.

\begin{abstract}
Increased interest in shared virtual environments has resulted in a necessity to investigate different factors that influence user interaction, both with the environment and other users. Introducing different types of joint activities into computer games can support a high level of realism and user engagement. This paper investigates two types of shared work: collaboration - where users individually perform actions in the environment towards a common goal, and cooperation - where users simultaneously act on the same objects in a shared environment. A basic computer game which requires joint work between all players was developed and used as a case study. We analyzed user performance and enjoyment in order to see which type of shared work is more preferable. Furthermore, the influence of user expertise on their enjoyment was also checked. From this particular study, we conclude that participants prefer cooperation over collaboration, as it provides more active and realistic performance. User expertise does not significantly influence enjoyment in this type of shared work, indicating that inexperienced players can enjoy the game equally as the experienced.
\end{abstract}

\section{INTRODUCTION AND RELATED WORK}

Collaboration at a distance has become an important topic within the research community. It gained interest due to the growing popularity of virtual communities (e.g. SecondLife ${ }^{1}$ ) and Massive Multiplayer Online Role Playing Games (e.g. World of War$\left.\mathrm{craft}^{2}\right)$. Their success lies beyond advanced threedimensional (3D) graphics and immersion, in providing the possibility to interact and play with other players, either as a team or against each other. While most interaction in these applications is limited to communication and navigation, collaboration on closelycoupled tasks (Otto et al., 2006), - where the actions of collaborating people are directly depending on each other, - has received less focus.

Three levels of shared work are defined in virtual environments (VE) (Ruddle et al., 2002). The first level defines collaboration as an ability to com-

\footnotetext{
${ }^{1} \mathrm{http}: / / \mathrm{www}$. secondlife.com

${ }^{2} \mathrm{http}: / /$ www.worldofwarcraft.com
}

municate and perceive the shared environment. The second level extends this ability, allowing individual modification of the environment. The third one is defined by simultaneous impact of multiple users on the same objects or parts of the environment (e.g. shared object manipulation). The last one is also often called cooperation (Broll, 1995), and this term will be used throughout the paper to refer to the third type of shared work. While the first and the second levels are actively used for most existing 3D multi-user computer games (for example, World of Warcraft), where people co-exist and interact in order to achieve a shared goal, we assume exactly the third type of collaboration can increase user engagement and add more realism to the virtual performance. One of the recent games that has become popular quickly and that supports the third type of collaboration is Little Big Planet ${ }^{3}$, where players encounter a shared challenge or obstacle, and often have to manipulate the same object simultaneously. Such popularity triggers a necessity to fully assess the potential of coopera-

\footnotetext{
${ }^{3}$ http://www.littlebigplanet.com
} 
tive activities for 3D computer games. Further possible advantages over collaboration based on individual performance have to be investigated as well in order to provide players with an enjoyable game experience.

There is a substantial amount of work investigating different aspects of collaboration and cooperation within small groups. Although the research is not always related to computer games, we believe that most of the findings can be applied in the context of game development. The major part of research in this area focuses on the implementation of collaborative and cooperative interaction techniques (Bowman et al., 2008), such as shared object manipulation. For example, Pinho et al. (Pinho et al., 2008) present a framework that supports development of cooperative manipulation techniques. Another cooperative manipulation technique called "SkeweR", that allows two users to move the same virtual object simultaneously in a VE, is presented in (Duval et al., 2006). Besides interaction techniques, several researches covered such issues like providing correct perspective views over the shared environment (Provenzano et al., 2007) and support for various types of feedback (Ullah et al., 2009).

Also, several studies focus on perceptional aspects of collaboration and cooperation, namely presence and co-presence within shared virtual environments (Schroeder et al., 2001; Heldal et al., 2005). These studies investigate factors that contribute to effective collaboration and people's behavior in distributed VEs while performing closely-coupled tasks. Similar work performed by Roberts et al. (Roberts et al., 2003) focuses on concurrent interaction with shared objects by users of a variety of display system configurations.

While most existing research focuses either on collaboration (Heldal et al., 2005) or cooperation (Roberts et al., 2003) separately, we realize the importance of their comparison. The contribution of our work is the comparative analysis of collaborative and cooperative activities, based on users' behavior and preferences while playing a multi-user 3D computer game.

In following section, we describe the conducted experiment. Section 3 presents the results of the user experiment, which are discussed and concluded in Section 4.

\section{EXPERIMENT DESCRIPTION}

In order to compare collaboration and cooperation, and more specifically to assess the influence of different factors on users' enjoyment, an experiment was conducted. The purpose of this experiment was to determine the factors that have a positive impact on interaction in a shared virtual environment for collaboration and cooperation. We wanted to see which setup is found to be more enjoyable and engaging by users in order to provide some guidelines for further application development (e.g. games), where this particular type of joint work can be beneficial.

We believe that cooperative activities, although not always easy and straightforward to perform, will increase enjoyment of users' interaction and provide a more convincing shared experience of working together. We expect therefore cooperative activities to be more preferable for most participants based on several criteria (i.e. performance, how natural the interaction was, etc.) and provide more enjoyable interaction among partners when compared to the collaborative condition.

During the experiment ten groups of three people were sequentially asked to play a basic 3D game with the shared goal of collecting 20 digits in the environment together with two partners. The game was represented as an environment that consists of two virtual houses containing 20 cubes (figure 1). Half of these cubes had a digit on one of their sides. In order to complete the game participants were asked to calculate the sum of all digits, and then to select the correct number (corresponding to the calculated sum) out of four options, presented as a four-item menu. To explore the environment, participants had to select and manipulate objects.

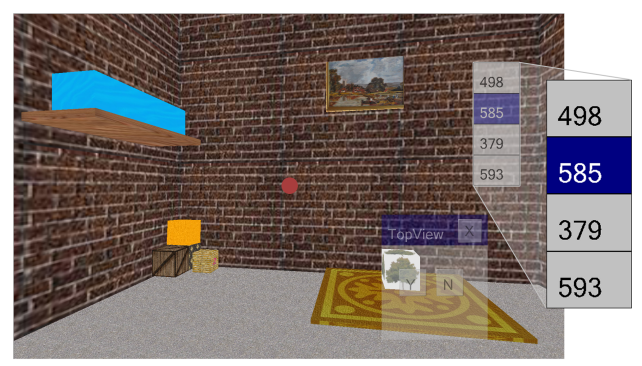

Figure 1: The task.

The experiment consisted of 2 sessions: collaborative and cooperative. For every session a different environment was created with the same level of complexity. In the collaborative session every object could be moved or rotated by a single person. In the cooperative session there were two types of objects: those that can be manipulated by one user and those that required joint manipulation by two users. If an object of the second type was selected, a help message was generated to inform other participants within the same house in the virtual environment that there is a person requiring a partner's help. However, the mes- 
sage did not indicate who triggered the call for help and where that person was located. Participants had to discuss this event to discover the feasibility to help each other. If none of the co-players was in the same house, the person who initially selected such an object got another message, indicating that his/her partners were not in the same area.

To indicate successful object selection visual feedback was provided. The object highlighted in red indicated it is already selected but in order to manipulate it two people are required. The object highlighted in green indicated that it is selected and can be manipulated. To select, manipulate and deselect an object the SpaceMouse or the SpaceExplorer was used. To move or rotate object jointly with a partner, participants had to simultaneously move an object in the same direction using their devices.

Although we designed this game to be played remotely, for observational reasons, participants were present in the same room (as shown in figure 2). During the experiment participants were allowed to communicate. Due to the co-located setup participants were able to talk to each other without using any communication technology (e.g. Skype). Because every participant was focused on actions happening on his monitor, other communication channels of real life (facial expressions, gestures, etc.) were not taken into account. Apart from voice communication they had a text messenger running on their laptops which was used to share already found digits to the partners. Once all 20 digits were found, one of the group members calculated their sum and selected the answer from the four-item menu (figure 1). Participants were supervised in order to avoid guessing the correct answer from the menu.

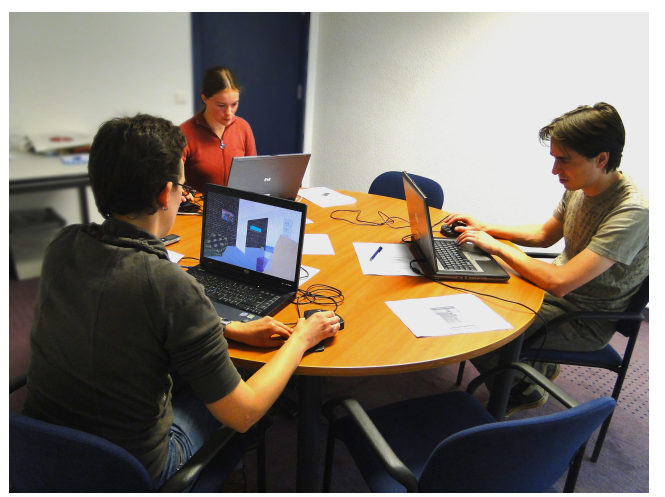

Figure 2: The experiment setup.

The time to complete each session was captured. After each session participants were asked to complete an individual questionnaire regarding their experience. In this questionnaire participants had to evaluate their performance and preference for every condition on a 10-point likert scale. Questions were grouped into several categories: enjoyment, level of working together, performance and communication activity. Additionally, we asked participants to indicate the level of their expertise in collaborative virtual environments.

\section{RESULTS}

The main goal of the conducted experiment was to compare two levels of shared work: collaboration and cooperation. Moreover, we present an analysis that shows the influence of the users' expertise on their performance and enjoyment. Finally, we analyze users' behavior based on observations. The comparative analysis is performed using paired-samples t-tests.

\subsection{Comparison of Collaboration and Cooperation}

\subsubsection{Task Completion Time}

The analysis has shown that it took significantly longer for participants to complete the task in the cooperative setup $(t(9)=-3.867, p=0.004)$. Figure 3 shows the task completion time for every group in both conditions.

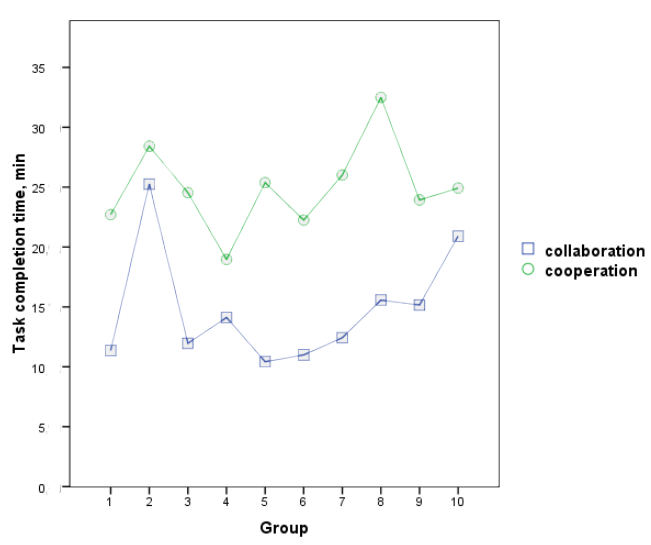

Figure 3: Task completion time for collaboration and cooperation.

As long task completion time may cause boredom and fatigue, it is important to investigate the effect of the length of the task on the overall enjoyment of users. We analyzed both types of joint work in order to search for any correlation with the time. We found a significant negative correlation $\left(R^{2}=-0.687\right.$, $p=0.028$ ) between enjoyment of collaboration and 
total time. For the cooperative condition no correlation was found between task completion time and enjoyment, indicating that people enjoy interaction even if more time to complete the task was required. This result has an important impact for game development, where it is important to engage players for a longer period of time. Nevertheless, it is still necessary to investigate the influence of time on users' enjoyment for continuous long-term gaming.

From observations we discovered that cooperation took longer because it was often necessary to wait for the partner in order to perform the joint activity. It also took some time to find the location of a person who needed help in the virtual environment. Taking into account these findings we can conclude that completion time can be considered as a task-dependent variable, but users still enjoy longer interaction when they are cooperating with their partners.

\subsubsection{Enjoyment and Performance}

Participants had to rate two types of shared work from different perspectives. We collected responses of individuals regarding their work under both conditions. All questions were grouped into several categories which were analyzed separately.

In table 1 all statistics are given in order to compare the collaborative and cooperative conditions, based on the individual responses. They are grouped similarly to the categories of the questionnaire given to participants. As we can see, participants enjoyed the cooperative task significantly more. They performed almost equally active as no difference was found across the conditions. Furthermore, we additionally asked them to evaluate their group activity in the collaborative and cooperative settings. We found that as a group they performed significantly more active during the cooperation $(t(9)=-3.250$, $p=0.01$ ). This can be explained by the nature of the given task as in a cooperative setting participants are more dependent on their co-players.

Manipulation of objects during the cooperative task required more effort and cohesive work from participants, and was indicated to be significantly harder than single-user object manipulation. But, as indicated before, such difficulty did not significantly influence the overall user enjoyment of cooperation.

Another question that was asked related to users' level of working together with others, rather than on their own. Responses showed another advantage of cooperative work - it provides a significantly higher feeling of working together as a group comparing to the one in collaboration. Furthermore, participants also indicated the cooperative condition as a more natural way of working together as a team. We also found that there was no significant difference across the two conditions regarding the level of awareness that participants perceived, indicating that they were equally aware of the situation in the environment during their activity.

The activity of communication and the level of its importance were also analyzed. As mentioned before, cooperation required much more communication and coordination from participants. Statistical analysis has shown that individuals communicated significantly more when they cooperated. Users also indicated that communication was more important for this session. They said that the collaboration session "did not require much communication, and the task itself can be actually completed without communication", while for the cooperative session "it would be impossible to complete the task if the communication is prohibited".

\subsection{Influence of User Expertise on Shared Work}

We asked participants to define their level of expertise in 3D single-user $(M=5.2, S D=2.93)$ and multiuser $(M=3.67, S D=2.43)$ virtual environments. Although most participants did not have much experience in shared virtual activities they still enjoy playing the game under both conditions. Moreover, we found no correlation between expertise level and enjoyment. This indicates that both experienced and less experienced users can equally enjoy activities with others in multi-user virtual environments. Additionally, no influence of user expertise on completion time was found.

Test groups were randomly formed, so that certain groups contained only inexperienced or only experienced users, or were mixed. That is why we additionally compared group enjoyment and average experience within each group. We found no significant influence of the experience on the group enjoyment. In spite of such heterogeneity, user interaction during both sessions was enjoyable for every participant.

Additionally, we found no correlation between $e x$ pertise and level of working together, which proves the fact that despite lack of experience users got an equally high perception level of working together with their collaborators for both conditions.

\subsection{Users' Behavior}

Previous sections already partially covered our analysis of users' behavior. Further analysis of user observations reveals interesting information regarding the users' experience. This section presents the collected 
Table 1: Comparison of collaboration and cooperation based on individual responses.

\begin{tabular}{|c|c|c|c|}
\hline Factor & \multicolumn{4}{|c|}{ Responses } \\
\hline Enjoyment & $t(29)=-2.421, p=0.022$ & $M_{\text {coll }}=6.37, S D_{\text {coll }}=1.5$ & $M_{\text {coop }}=7.23, S D_{\text {coop }}=1.52$ \\
\hline Activity & $t(29)=-0.711, p>0.05$ & $M_{\text {coll }}=7.43, S D_{\text {coll }}=1.3$ & $M_{\text {coop }}=7.63, S D_{\text {coop }}=1.3$ \\
\hline Object manipulation & $t(29)=2.079, p=0.047$ & $M_{\text {coll }}=7.1, S D_{\text {coll }}=1.9$ & $M_{\text {coop }}=6.3, S D_{\text {coop }}=1.37$ \\
\hline \multicolumn{5}{|c|}{ Working with others } \\
\hline Level of togetherness & $t(29)=7.623, p=0.000$ & $M_{\text {coll }}=5.33, S D_{\text {coll }}=2.12$ & $M_{\text {coop }}=8.07, S D_{\text {coop }}=1.23$ \\
\hline Naturalness & $t(29)=-3.713, p=0.01$ & $M_{\text {coll }}=6.44, S D_{\text {coll }}=1.38$ & $M_{\text {coop }}=7.37, S D_{\text {coop }}=1.45$ \\
\hline Awareness & $t(29)=-1.925, p>0.05$ & $M_{\text {coll }}=5.87, S D_{\text {coll }}=1.63$ & $M_{\text {coop }}=6.43, S D_{\text {coop }}=1.70$ \\
\hline \multicolumn{5}{|c|}{ Communication } \\
\hline Activity & $t(29)=-5.835, p=0.000$ & $M_{\text {coll }}=5.77, S D_{\text {coll }}=1.87$ & $M_{\text {coop }}=7.87, S D_{\text {coop }}=1.079$ \\
\hline Importance & $t(29)=-9.380, p=0.05$ & $M_{\text {coll }}=5.1, S D_{\text {coll }}=1.73$ & $M_{\text {coop }}=8.33, S D_{\text {coop }}=1.06$ \\
\hline
\end{tabular}

and analyzed qualitative information concerning the users' experience while collaborating and cooperating.

Preferences The analysis shows that participants preferred the cooperative session significantly more than the collaborative one, rating their enjoyment higher. Observing people playing the two games allows us to draw a conclusion what actually caused such enjoyment.

One of the main reasons why people liked both conditions is the collaborative nature of the games. The fact that they were not single players had a positive impact on their overall experience. Collaboration was considered less interesting and less fun compared to the cooperative session because of the reduced interaction. Participants performed more individually, rather than as a group to complete the task. Some players said they did not felt part of a group, but rather co-existed with others.

Cooperation involved more activity in order to complete the game, resulting in a high level of interaction with other participants. Although it was often necessary to wait for a helper, participants still were excited to perform joint activities. Moreover, frustration caused by different views on the same objects, did not influence the enjoyment. Almost all players experienced more fun when playing the cooperative game, as it really gave the feeling of team work. People pointed out that "being fun" is more crucial than "being difficult" when playing computer games.

Voice communication In our previous work (Beznosyk et al., 2010) we demonstrated that the inclusion of communication was significantly important for collaborative tasks in the virtual environments. In the current experiment, voice communication was included for both the collaborative and the cooperative sessions. We wanted to determine when it was necessary to talk with the partners, and what kind of communication was used while working under different conditions.

First of all, we noticed that participants who had difficulties with the actual performance (e.g. object manipulation) used communication to compensate their skills. When it was too hard to manipulate an object, they became more active in a group discussion. They communicated actively during strategy discussion and along the task itself. During the collaborative session, communication was mainly used for strategical discussions at the beginning, to do a bit of joking or to keep others aware what the participants were doing. Communication was indicated as the only instrument of collaboration, as players performed individually rather than as a team. We also observed that a few pairs did not even discuss even their strategy, which resulted in longer task completion time. They concluded that it was not necessary to communicate, because they were able to see if there is anyone around.

During the cooperative session, all pairs admitted the necessity of communication. Players not only talked about their strategy or role division among the group members, but also coordinated their movements actively. They indicated that the help message they saw, when someone picked up an object which had to be manipulated jointly, does not really help if you cannot communicate about the object description and location. For the cooperative condition the communication was almost continuous, due to someone needing help. Vocal conversation was also used as an awareness mechanism in the same way as for collaboration. People communicated regarding their positions and actions in the virtual environment, thus making their performance smoother.

It is obviously preferable to include voice communication when working together with others. It contributes by making interaction more fluent and realistic. The main purpose of communication during the collaboration was division of the work across group members, which can also be replaced with a text chat. At the same time, cooperation required communication mainly for real-time coordination, which would be difficult to complete having only a text chat. 


\section{DISCUSSION AND CONCLUSION}

The results obtained during this experiment, confirming our initial assumptions, have an important impact for the implementation of shared 3D virtual environments, in particular for game development. Two types of joint work, collaboration and cooperation, were analyzed and compared in order to see which one is more preferred by players. We found that participants enjoyed cooperative tasks more, irrespective to the level of their expertise in $3 \mathrm{D}$ environments. We observed that non-experienced players were also actively involved in the task execution, and enjoyed it not less than more experienced partners. Cooperation also supported a higher level of working together. The main advantage was that players not only had a joint goal, that could be achieved concurrently, but they really had to perform activities together in order to reach the final aim.

Additionally, we found no negative effect of the longer task completion time of cooperative activities on the user enjoyment, which existed for collaboration. Therefore, we can assume that similar cooperative activities - where users can truly work together in a shared environment - will be beneficial in computer games. We do not reject collaborative activities as they are also found to be successful for gaming (Seif El-Nasr et al., 2010). Moreover, combining the two types of joint work within the same games has potential. We believe, that this will provide more dynamic interaction, as players will be able to switch their activities from one type to another.

These results prove that incorporating such activities into computer games will increase users' enjoyment and engagement, providing truly shared experience. But the implementation of cooperative systems for multi-user computer games requires more effort from developers. Further research is necessary in order to develop collaborative and cooperative interaction techniques suitable for large groups, as well as fine-grained algorithms to keep interaction synchronized among all participants.

\section{ACKNOWLEDGEMENTS}

The research described in this paper is directly funded by Hasselt University BOF financing. The authors would like to thank Lode Vanacken for his assistance with the implementation and all participants who contributed to this research by taking part in the experiment.

\section{REFERENCES}

Beznosyk, A., Raymaekers, C., Coninx, K., Quax, P., and Lamotte, W. (2010). Investigating the influence of communication and input devices on collaboration in virtual environments. In Proc. GRAPP2010.

Bowman, D. A., Coquillart, S., Froehlich, B., Hirose, M., Kitamura, Y., Kiyokawa, K., and Stuerzlinger, W. (2008). 3d user interfaces: New directions and perspectives. IEEE Computer Graphics and Applications, 28:20-36.

Broll, W. (1995). Interacting in distributed collaborative virtual environments. In Proc. VRAIS 1995, pages 148155. IEEE.

Duval, T., Lecuyer, A., and Thomas, S. (2006). Skewer: a $3 \mathrm{~d}$ interaction technique for 2-user collaborative manipulation of objects in virtual environments. $3 D$ User Interfaces, pages 69-72.

Heldal, I., Steed, A., and Schroeder, R. (2005). Evaluating collaboration in distributed virtual environments for a puzzle-solving task. In Proc. HCII 2005.

Otto, O., Roberts, D., and Wolff, R. (2006). A review on effective closely-coupled collaboration using immersive cve's. In Proc. of ACM VRCIA 2006, pages 145-154. ACM.

Pinho, M. S., Bowman, D. A., and Freitas, C. M. (2008). Cooperative object manipulation in collaborative virtual environments. J. Braz. Comp. Soc., 14(2):54-67.

Provenzano, L., Delzons, J., Plnacoste, P., and Vandromme, J. (2007). Designing viewpoint awareness for $3 \mathrm{~d}$ collaborative virtual environment focused on real-time manipulation of multiple shared objects. In Virtual Reality, volume 4563 of $L N C S$, pages 147-156. Springer Berlin / Heidelberg.

Roberts, D., Wolff, R., Otto, O., and Steed, A. (2003). Constructing a gazebo: supporting teamwork in a tightly coupled, distributed task in virtual reality. Presence: Teleoper. Virtual Environ., 12(6):644-657.

Ruddle, R. A., Savage, J. C. D., and Jones, D. M. (2002). Symmetric and asymmetric action integration during cooperative object manipulation in virtual environments. ACM Trans. Comput.-Hum. Interact., 9(4):285-308.

Schroeder, R., Steed, A., Axelsson, A.-S., Heldal, I., Åsa Abelin, Wideström, J., Nilsson, A., and Slater, M. (2001). Collaborating in networked immersive spaces: as good as being there together? Computers \& Graphics, 25(5):781-788.

Seif El-Nasr, M., Aghabeigi, B., Milam, D., Erfani, M., Lameman, B., Maygoli, H., and Mah, S. (2010). Understanding and evaluating cooperative games. In Proc. CHI 2010, pages 253-262. ACM.

Ullah, S., Otmane, S., Richard, P., and Mallem, M. (2009). Human performance in cooperative virtual environments : the effect of visual aids and oral communication. IJVR, 8(4):79-86. 